\title{
Taurine Reverses Oxidative Damages and Restores the Muscle Function in Overuse of Exercised Muscle
}

\author{
Anand Thirupathi'*, Ricardo A. Pinho², Julien S. Baker ${ }^{3}$, Bíró István ${ }^{4}$ and Yaodong Gu* \\ ${ }^{1}$ Faculty of Sports Science, Ningbo University, Ningbo, China, ${ }^{2}$ Laboratory of Exercise Biochemistry in Health, Graduate \\ Program in Health Sciences, School of Medicine, Pontifícia Universidade Católica do Paraná, Curitiba, Brazil, ${ }^{3}$ Department \\ of Sport, Physical Education and Health, Hong Kong Baptist University, Hong Kong, China, ${ }^{4}$ Faculty of Engineering, \\ University of Szeged, Szeged, Hungary
}

\section{OPEN ACCESS}

Edited by:

Mohsin Khan,

Temple University, United States

Reviewed by:

Song-Gyu Ra,

Tokushima University, Japan

Erik Alexander Blackwood,

San Diego State University Research

Foundation, United States

${ }^{*}$ Correspondence:

Anand Thirupath ananthzea/@gmail.com

Yaodong Gu

guyaodong@hotmail.com

Specialty section:

This article was submitted to

Oxidant Physiology,

a section of the journal

Frontiers in Physiology

Received: 12 July 2020

Accepted: 26 August 2020

Published: 26 October 2020

Citation:

Thirupathi A, Pinho RA, Baker JS, István B and Gu Y (2020) Taurine

Reverses Oxidative Damages and Restores the Muscle Function

in Overuse of Exercised Muscle.

Front. Physiol. 11:582449.

doi: 10.3389/fphys.2020.582449
Exercise-induced oxidative stress is linked with the expression level of endogenous antioxidants, but these antioxidants cannot overcome all oxidative stress-related damages in the cells, particularly when cells are under physiological stress. Sometimes, compounds are needed for cellular function, which are produced/activated within the cells, and these compounds can be synthesized by performing exercise, especially high-performance exercise. Taurine is a sulfur-containing amino acid used for various physiological functions. However, its synthesis and accumulation under the oxidative environment may be compromised. Recently, we have shown that taurine level is increased during exercise performance with a decrease in oxidative damage in overused muscles. Other studies have also shown that short-term supplementation with taurine increased physiological performance during severe work intensities, suggesting the role of taurine in improving muscle performance during exercise. However, its precursor cysteine is used in the synthesis of other compounds like GSH and Coenzyme A, which are important for regulating the redox system and energy homeostasis. It is, therefore, important to understand whether taurine synthesis within the cells can blunt the activity of other compounds that are beneficial in preventing oxidative damage during intense exercise. Furthermore, it is important to understand whether taurine supplementation can prevent the conditions observed in the physiological stress of muscles. This review discusses how taurine synthesis could alter exercise-induced ROS generation and the relationship between the physiological stress of muscle and subsequent improvements in exercise performance.

Keywords: taurine, exercise, muscle overuse, oxidative stress, antioxidants

\section{INTRODUCTION}

Skeletal muscle is one of the most specialized tissues with plasticity in the human body. It accounts for approximately $40-50 \%$ of the total body mass, and it can easily withstand different stimuli from exercise-induced mechanical stress and metabolic stress to other environmental stimuli, including heat exposure and nutrient availability (Rivas and Fielding, 2012; Frontera and Ochala, 2015). These adaptions are linked with various complex systemic interactions such as cellular response, oxidative capacity, and regulation of gene expression and protein level, and this could be achieved through various signaling and structural adaptions (Suhr et al., 2013). Physical exercise is one of the factors that positively promotes these skeletal muscle adaptations. However, strenuous exercise 
may affect various biochemical and myocellular alterations. For example, high-intensity exercise-induced muscle lesions, following muscle impairment, and muscle adaption. The reasons for muscle overuse injury also include cumulative trauma, which is related to volume and overload intensity or repetitive use and muscle stress.

Reactive oxygen species (ROS) play a crucial role in the exercise-induced pathophysiology of muscle, but it occurs in an exercise-dependent manner (Thirupathi and Pinho, 2018). Moderate exercise is believed to produce a small amount of ROS that is involved in regulating muscular adaption during exercise. However, increased contractile activity of skeletal muscle increases ROS generation which causes various physiological and biochemical adaptions such as mitochondrial biogenesis and changes in active myofibres. ROS is a core factor in regulating both cellular and physiological functions by affecting various signaling or acting as a signaling molecule. Our group has also demonstrated that ROS generation and oxidative stress are involved in trauma-induced muscle injury (Silveira et al., 2013). Further to this, muscle damage and subsequent repair are accompanied by the recruiting of phagocytic cells and macrophages which are additional sources of ROS generation leading to damage of the musculature itself and muscle impairment.

Muscle-induced oxidative damage is often unavoidable and requires therapeutic interventions, e.g., supplementary foods containing antioxidants, that regulate the redox process (Waters et al., 2010; Molnár et al., 2016). However, some of these supplements may reduce the beneficial effects of exogenous antioxidants during strenuous exercise, and selecting a proper supplementary nutrient is necessary to maintain muscle performance (Harty et al., 2019). Taurine is one such supplement that enhances cellular functions in several ways, e.g., stabilizing the membranes and regulating the cell volume, balancing redox homeostasis, and controlling ion channels (Schaffer et al., 2014; De Luca et al., 2015; Lambert et al., 2015). In addition, taurine plays a significant role in reducing the oxidative stress and inflammatory responses induced by exercise. Taurine is a sulfur-containing free amino acid present in the body with concentration ranges between 5 and $20 \mu \mathrm{m} / \mathrm{g}$, particularly in excitable tissues such as muscles, but it can also be obtained from external sources like meat and sea-food (Huxtable, 1992, 2000; Schaffer et al., 2010). Studies have shown that overuse of muscle depletes taurine level and the loss of taurine occurs mostly in the fast-twitch fibers (Dawson et al., 2000; Matsuzaki et al., 2002; Yatabe et al., 2003). Therefore, taurine supplements are necessary to compensate for intracellular taurine levels and to increase exercise performance. However, the duration of taurine administration under chronic exercise conditions is yet to be established.

\section{Muscle Overuse and Synthesis of Taurine Under ROS Environment}

Muscle overuse affects taurine synthesis by producing ROS, and consequently, taurine-induced benefits are compromised. Taurine biosynthesis is achieved as a result of the following biochemical reactions: oxidation of cysteine by cysteine dioxygenase to form cysteine sulfinic acid; followed by decarboxylation of cysteine sulfinic acid by the cysteine sulfinic acid decarboxylase producing hypotaurine, and finally the oxidation of hypotaurine to taurine. Exercise-induced ROS generation can alter this sequence (Huxtable, 1992; Faggiano et al., 2005). As cysteine is a less abundant amino acid, its utilization for protein and non-protein synthesis can compromise taurine synthesis. However, exercise-induced ROS generation may primarily attack the sulfhydryl $(-\mathrm{SH})$ terminal of cysteine, which is the location where several cellular events are organized for the synthesis of proteins including taurine (Kolossov et al., 2015), and thus exercise-induced ROS is an important factor for organizing the above-mentioned scenario (Figure 1). For example, muscle overuse-induced cysteine oxidation and its intermediate cysteine sulfinic acid oxidation result in a reduced level of taurine and glutathione synthesis (GSH), ultimately compromising the taurine-induced benefits in the muscle, such as influencing GSH level and glutathionylation (Bertolone et al., 2020). Glutathionylation caused inhibition of reversible cysteine oxidation is compromised, which may fail to protect from oxidative damage during muscle contraction (Qin et al., 2013). However, the level of ROS is regulated by exercise-induced adaptation in the cells which may prevent further ROS-induced damage (Scheele et al., 2009; Kawamura and Muraoka, 2018).

Exercise affects the taurine turnover (Dawson et al., 2002; Matsuzaki et al., 2002), but it also varies between person to person due to the amount of protein intake or protein synthesis. Exercise-induced ROS generation also influences the protein synthesis-related signaling (Vaanholt et al., 2008). Furthermore, several other intermediate compounds, such as folic acid and enzymes like methyl tetrahydrofolate reductase (MTHFR), influence the availability of cysteine and further taurine synthesis, which are all affected by exercise-induced ROS generation (De Luca et al., 2015). Since glutathione and taurine share the same precursor, taurine synthesis may affect GSH synthesis which can increase the oxidative damages induced by overuse of muscles. Retaining taurine concentrations at the cellular level is important for obtaining exercise-induced benefits and in preventing exercise-induced muscle damage.

\section{Does Taurine Regulate Mitochondrial ROS Production in Overused Muscle?}

Exercise endurance is proportional to the mitochondrial function (Hood et al., 2019) and the mitochondrial membrane potential is partially responsible for mitochondrial function, regulating the intracellular ionic charges (Thirupathi et al., 2018). Hansen et al. have shown that taurine supplements can regulate proper proton pumping without affecting mitochondrial matrix alkalinity (Hansen et al., 2010). We have also shown that taurine supplementation maintained mitochondrial membrane potential in the overuse of muscle (Thirupathi et al., 2018). Muscle overuse-induced mitochondrial ROS is also prevented by taurine supplementation. For example, ROS is produced in several ways in the mitochondria, and succinate oxidation is one of the ways 


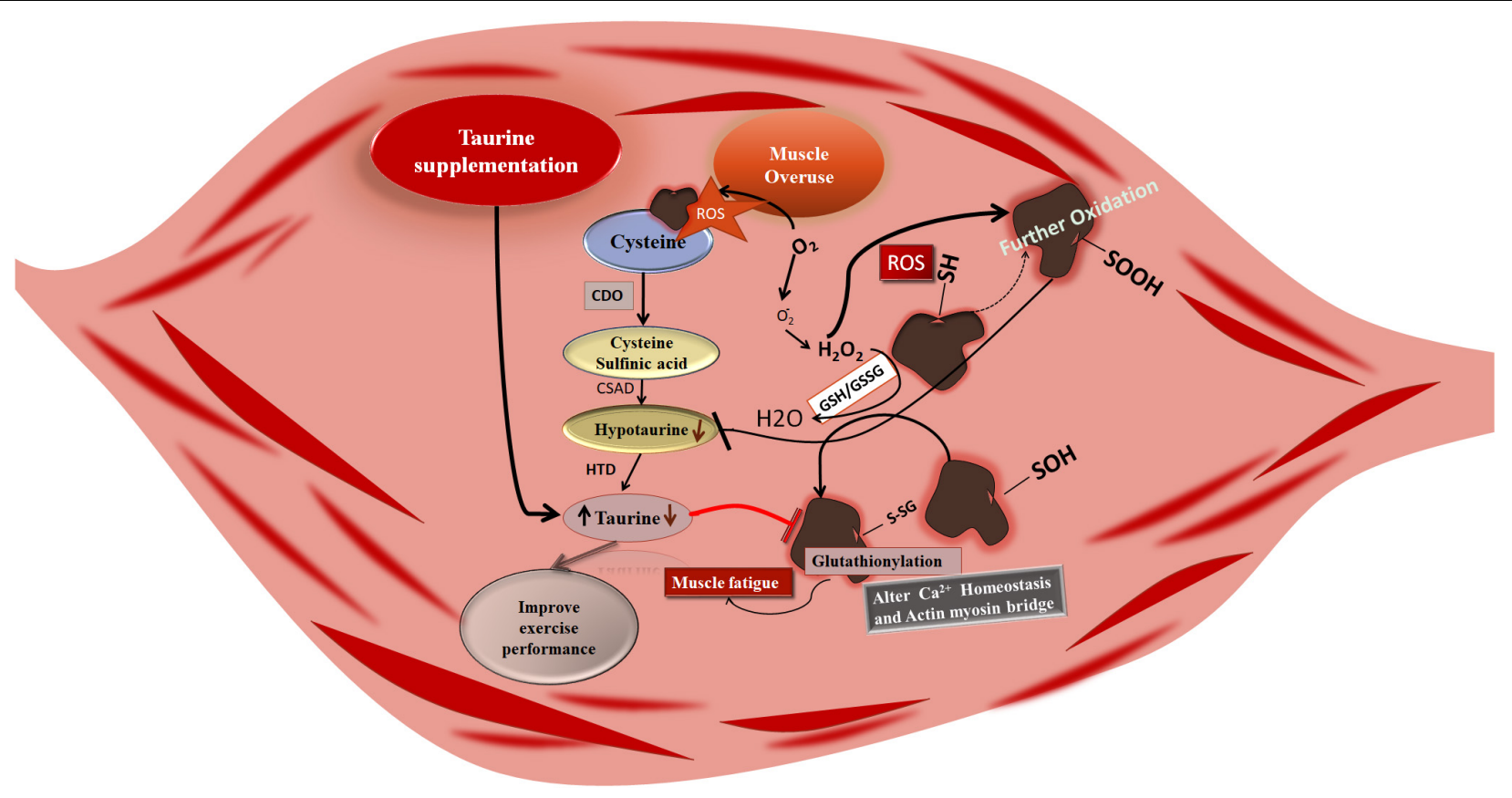

FIGURE 1 | Muscle overuse-induced cysteine oxidation and subsequent intermediary compound sulfinic acid oxidation compromising the synthesis of taurine and glutathione (GSH). Decreased levels of taurine and GSH may inhibit the glutathionylation resulting in reversible oxidation of cysteine. Further, disturbances in the glutathionylation may cause disturbances in the muscle function like $\mathrm{Ca}^{2+}$ dyshomeostasis and actin myosin bridge alteration which results in muscle fatigue; taurine supplementation increases the level of taurine in the muscle resulting in regulating $\mathrm{Ca}^{2+}$ homeostasis and actin myosin bridge activation by controlling ROS and improving exercise performance. CDO, cysteine dioxygenase; CSAD, cysteine sulfinic acid decarboxylase; HTD, hypotaurine dehydrogenase.

to generate ROS during NAD-linked substrates, such as coupled respiration with succinate in complex I via reverse electron transfer from complex II to I.

Our group has shown that taurine supplementation reduced ROS in overuse of muscles exposed to succinate, which produced $\mathrm{mtH}_{2} \mathrm{O}_{2}$ through regulating mitochondrial membrane potential and preventing the formation of semiubiquinone radical (Thirupathi et al., 2018). However, taurine does not increase the activity of other complexes except complex I in the ETC in overuse muscle, suggesting that taurine prevents ROS production at the complex I level (Figure 2). Overused muscles demand energy levels that are as high as possible at the initial level of onset of exercise to overcome strenuous exerciseinduced energy deprivation. This results in increased complex I activity and ultimately produces ROS due to possible electron flow blockage and reduction in the ubiquinone pool. This limits ATP production, and consequently reduces the performance of exercise and causes other dysfunctions in the muscles (Brand, 2016). Taurine can change this scenario at the complex I level to improve muscle function and exercise performance.

\section{Does Taurine Prevent DNA Damage in Overused Muscle?}

Muscle overuse releases a higher level of ROS and alters inflammatory cascades which ultimately leads to DNA damage and reduces DNA repair protein expressions. This can be prevented by long-term exercise by obtaining cellular adaptation
(Neubauer et al., 2008; Soares et al., 2015). Although studies have shown the effect of taurine on regulating inflammatory processes and ROS generation (Lin et al., 2013; Galan et al., 2018), taurine's role in reducing DNA damage is poorly understood particularly in the exercise overuse situation. We have shown that taurine administration at $150 \mathrm{mg} / \mathrm{kg}$ body weight decreased the DNA damage in overused muscles (Thirupathi et al., 2018). Taurine prevented the exercise-induced nitrosative inflammation and DNA damage through the NF-kB signaling pathway (Sugiura et al., 2013). Drug-induced taurine deficiency may alter several signaling activation such as the PI 3-kinase/AKT pathway that is linked with DNA damage-induced cell death (Pastukh et al., 2005). Hypoxic state-induced DNA damage is a common phenomenon, especially during muscle overuse. This may be linked with a low level of $\mathrm{Ca}^{2+}$ (Zhang et al., 2004; Husain and Mahmood, 2020). Taurine's role against hypoxic injury is achieved through regulating $\mathrm{Ca}^{2+}$ homeostasis (Schaffer et al., 2002). Therefore, taurine could be a potential candidate for preventing ROS-induced DNA damage in the overused muscle.

\section{The Role of Taurine in the Muscle Phenotype Under Exercise Conditions}

Taurine increases the muscle level, and this could be due to phenotypic-specific contractile properties (De Luca et al., 2015; Terrill et al., 2016). Supplementation of taurine with a higher content of amino acid in the skeletal muscle leads to an increase in muscle chloride channel conductance $(\mathrm{gCl})$. 


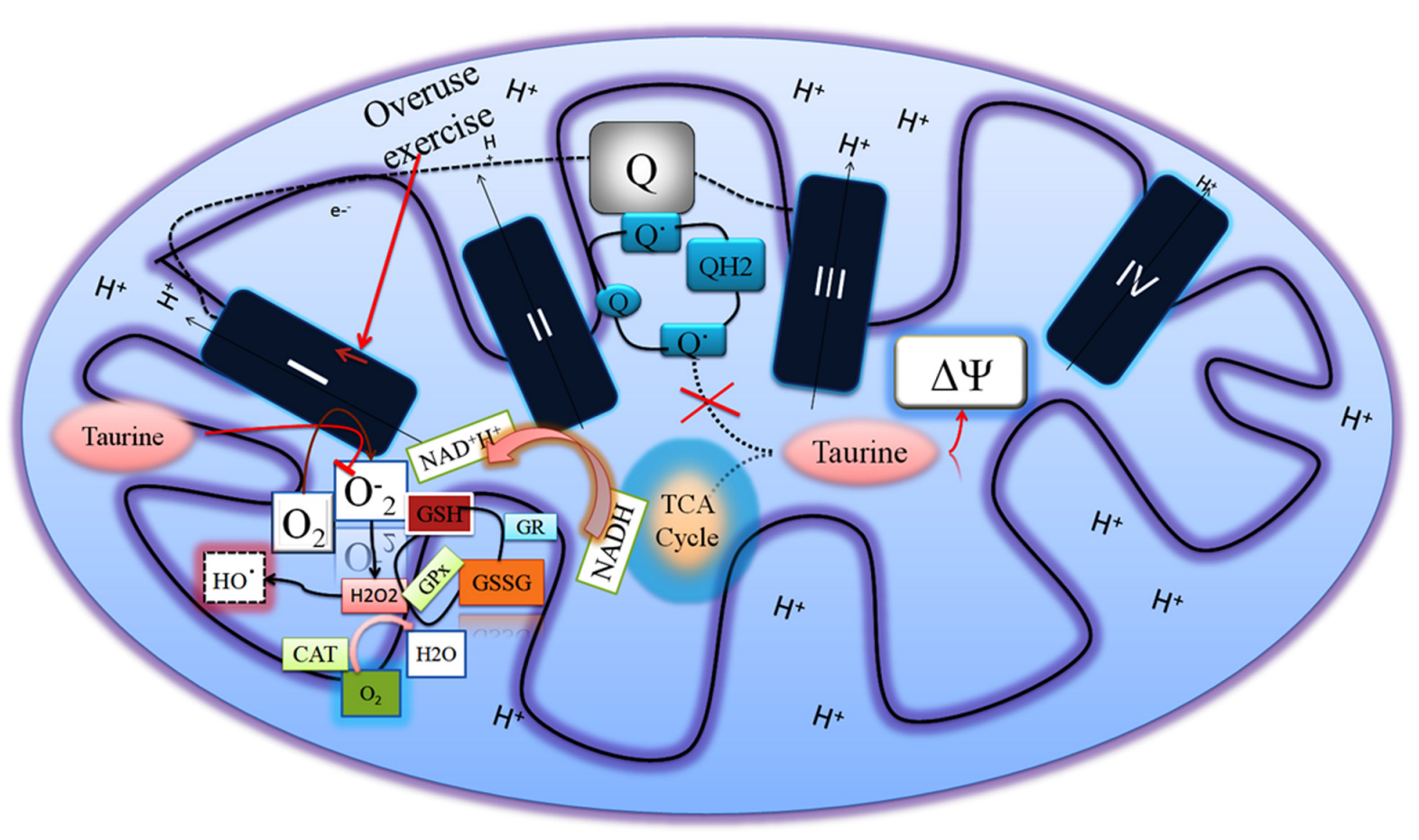

FIGURE 2 | Overused muscles increase the complex I activity to compensate for energy deprivation, which increases superoxide radical generation. Taurine regulates the mitochondrial membrane potential and decreases both the superoxide radical in the complex I and semiubiquinone radical so as to increase the muscle performance.

Taurine transporter (TauT) expression during myogenesis is increased, and taurine stimulates the myofibre differentiation (Uozumi et al., 2006; Miyazaki et al., 2013). Although these studies showed the importance of taurine in muscle phenotype, the mechanism that regulates the muscle phenotype is unclear. The possible mechanism that regulates the muscle phenotype is mitochondrial biogenesis and its role in tissue development (De Luca et al., 2015). Taurine could protect the susceptibility of exercise-induced muscle damage by controlling oxidative stress and inflammation. Stress-induced muscle damage and subsequent taurine treatment increased the susceptibility to muscle damage by up to $40 \%$ or more (Terrill et al., 2016; Ra et al., 2016; Waldron et al., 2019). However, taurine could not protect the muscle damage in the initial stretching, and it can protect the subsequent pathology, which is associated with initial injury through regulating inflammatory response and oxidative stress. Additional studies are required in order to find a taurine induced specific mechanism that supports to protect exercise-induced muscle damage and necrosis.

The overuse of muscle alters muscle proliferation and regeneration capacity, and several muscle-specific proteins are involved in regulating the muscle phenotype, including $\mathrm{MyoD}$, Myf5, and myogenin (Flann et al., 2011; Baumert et al., 2016). Exercise is believed to activate these muscle-specific proteins to regulate the muscle phenotype, however, how these proteins are organized to reverse the overuse injury in muscle into normal is ambiguous. Muscle overuse-induced ROS could be the main contributory factor that alters the muscle regulatory protein expression, compromising the muscle function. Taurine can alter these scenarios by regulating calcium uptake and inflammatory mediators and maintaining optimal ROS generation (Miyazaki et al., 2013; Nam et al., 2017). Increased ROS generation facilitates various adaptation in the muscle cells for increasing force generation, and its concentration yet to be determined and is one of the limitations of the ROS- induced benefits (Radák et al., 2001; Thirupathi et al., 2018, 2020). We recently demonstrated that taurine-associated gold nanoparticle increased the Myf-5 protein in the overuse of the muscle to protect the muscles from further damage, suggesting that taurine associated gold nanoparticle may optimally maintain the ROS level so as to increase the ROSinduced benefits, such as regulating muscle regulatory proteins (De Luca et al., 2003; Warskulat et al., 2004; Goodman et al., 2009; Silva et al., 2011; da Silva et al., 2014; Horvath et al., 2016; Terrill et al., 2016). Furthermore, the induction of the Myf5 protein requires amino acid methylation, particularly arginine and cysteine, and ROS could mediate cysteine methylation, which ultimately affects the synthesis of taurine. How these mechanisms are orchestrated under conditions of muscle overuse is still unknown.

\section{Taurine's Role in Exercise Performance}

Taurine regulates several physiological functions. Since its discovery in ox bile in 1827, understanding of taurine's role in increasing exercise performance is limited. Studies have shown that the administration of taurine has a significant effect on exercise performance, but other studies have demonstrated that 
taurine did not influence exercise performance. For example, $1 \mathrm{~g}$ of taurine administration improved the $3-\mathrm{km}$ time trail running before the exercise, whereas during the 4-km running trail did not have a significant effect on exercise performance with taurine supplementation (Balshaw et al., 2013; Ward et al., 2016). This may be due to the exhausting performance increasing ROS generation at a threshold activity, and further work on the effects of taurine and the duration and intensity of exercise need to be established. Therefore, a decrease in muscle contraction and relaxation and experimental protocol using correct dosages of taurine may optimally maintain the ROS generation in the muscle cells, which ultimately improves exercise performance. Compounds such as GSH and coenzyme A may reduce the benefits of taurine, as they are utilized to synthesis other compounds. Keeping optimal taurine levels in the muscle cell is difficult, and facilitating taurine synthesis in the cells may advance the role of ROS-induced benefits through taurine synthesis instead of ROS-induced muscle damage (De Luca et al., 2003; Ito et al., 2010; McLeay et al., 2017).

The effect of the duration of taurine administration in increasing exercise performance is ambiguous. For example, oral administration of taurine may promote the exercise performance initially, but long-term exercise using taurine does not have a significant effect, suggesting that oral intake of taurine at the initial level may promote the exercise performance, but longer duration of exercise may not retain the cellular taurine levels (Rahman et al., 2011; De Carvalho et al., 2017; Sajid et al., 2017; Niu et al., 2018; Li et al., 2019; Seidel et al., 2019). However, strenuous exercise-induced muscle damage may be reversed and the duration of taurine administration and effects on chronic exercise conditions need to be established to evaluate the total beneficial effects of taurine supplementation.

Exercise training in terms of type, duration, and intensity perturbs the oxidative stress status and amino acid pool, including taurine in athletes. This demands additional supplements to improve exercise performance. Studies have shown that taurine supplementation improved the exercise performance, and the mechanism that is involved to improve the muscle performance is following: (I) increased concentrations of taurine in the plasma increase the calcium uptake and release to contractile filaments, which further enhances the force production; (II) regulating muscle membrane; and (III) increasing the mitochondrial buffering (El Idrissi, 2008; Galloway et al., 2008; Batitucci et al., 2018). Speed and intensity during exercise may be achieved through an increased level of taurine, which may indicate its release into muscle fibres (Ward et al., 1999). The nature of the increase in taurine concentration in the plasma content after strenuous exercise must be established, as this may be the indication of muscle damage, muscle fatigue, or osmolarity adaptation within the blood (Ward et al., 1999). The release of taurine from the muscle and other blood constituents may disturb the level of taurine. For example, changes in the plasma osmolarity due to exercise can affect the distribution of taurine in the plasma, and plasma volume decreases postmarathon. Taurine and its corelease of water maintain plasma volume and may redistribute the taurine level to improve performance (Ward et al., 1999).

\section{Metabolic Action of Taurine in the Skeletal Muscle}

Taurine's metabolic action in maintaining normal physiological homeostasis may be related to its action on glucose uptake, insulin sensitivity, and further fat oxidation in the skeletal muscle. Studies have shown that taurine improved insulin sensitivity in the fructose-fed rat to stimulate glycolysis and gluconeogenesis (Mozaffari et al., 1986). Taurine deficiency showed an increased level of glucose uptake during treadmill running and glycolysis, resulting in elevated lactate production, which further contributed to impairing the exercise performance (Ito et al., 2014). As an energy sensor molecule, AMPK plays a crucial role in fatty acid oxidation and the peroxisome proliferator-activated receptor $\alpha(\operatorname{PPAR} \alpha)$, which regulates the oxidation and transport of fatty acids. AMPK-activated subunits and the level of PPAR $\alpha$ are reduced in the TauTKO muscle, suggesting that taurine is crucial for regulating energy metabolism during exercise in the skeletal muscle (Ito et al., 2014). Taurine supplementation may restore the energy homeostasis under pathological situations, whereas it improves muscle performance under normal conditions. For example, in one study, dietary supplementation with taurine attenuated the oxidative stress and inflammatory responses in patients with type 2 diabetes mellitus (T2DM) (Maleki et al., 2020).

\section{Effect of Taurine on Antioxidant Enzymes in the Skeletal Muscle}

Taurine affects the availability of antioxidant enzymes such as superoxide dismutase (SOD), catalase (CAT), and glutathione peroxidase (GPx), but its effect on antioxidant enzymes depends on its concentration. Taurine has increased the antioxidant enzyme activity in a dose-dependent manner in malathioninduced oxidative stress (Ince et al., 2017). It also formed taurine chloramine (TauCl), which may influence the availability of antioxidant enzymes in a dose-dependent manner. TauCl inhibits the overproduction of superoxide radicals in a dose-dependent manner (Kim and Cha, 2009). Studies have shown that $\mathrm{TauCl}$ activates the Kelch-like ECH-associated protein 1-nuclear factor E2-related factor 1 (Keap1-Nrf2) pathway (Sun Jang et al., 2009; Kim et al., 2010) and Nrf2 target genes including SOD, CAT, and heme oxygenase- 1 are activated. The taurine independently affects the Nrf1-ARE pathway affecting the antioxidant enzymes such as heme oxygenase-1. However, taurine supplementation does not influence the antioxidant enzyme level in the skeletal muscle following eccentric exercise. It can promote the synthesis of GSH, which results to increase the action of GPx, and this could be the mechanism of antioxidant defense (Silva et al., 2011; da Silva et al., 2014).

\section{CONCLUSION}

Muscle overuse may require a patient to take additional supplements, the function of which is to alter the inflammatory response and reduce oxidative stress without affecting muscle 
function. However, the duration of supplement administration must be established and monitored. Taurine reverses muscle function under overuse conditions in several ways, including by controlling mitochondrial ROS production, regulating membrane potential, preventing DNA damage, and inducing muscle regulatory proteins. ROS could be the mediator in organizing these conditions. Synthesis of taurine as a means of endogenously improving exercise performance is a major task. Optimal ROS generation due to exercise may facilitate the synthesis of taurine in various ways, such as by primarily attacking sulfhydryl (-SH) terminal of cysteine, the location where several cellular events are organized including the synthesis of taurine. However, how this is achieved through exercise-mediated ROS is yet to be established. Furthermore, the mechanism that drives ROS-induced effects in combination

\section{REFERENCES}

Balshaw, T. G., Bampouras, T. M., Barry, T. J., and Sparks, S. A. (2013). The effect of acute taurine ingestion on 3-km running performance in trained middle-distance runners. Amino Acids 44, 555-561. doi: 10.1007/s00726-0121372-1

Batitucci, G., Terrazas, S. I. B. M., Nóbrega, M. P., Carvalho, F. G. D., Papoti, M., Marchini, J. S., et al. (2018). Effects of taurine supplementation in elite swimmers performance. Motriz. Rev. Ed. Fís. 24:e1018137. doi: 10.1590/s19806574201800010011

Baumert, P., Lake, M. J., Stewart, C. E., Drust, B., and Erskine, R. M. (2016). Genetic variation and exercise-induced muscle damage: implications for athletic performance, injury and ageing. Eur. J. Appl. Physiol. 116, 1595-1625. doi: 10.1007/s00421-016-3411-1

Bertolone, L., Roy, M. K., Hay, A. M., Morrison, E. J., Stefanoni, D., Fu, X., et al. (2020). Impact of taurine on red blood cell metabolism and implications for blood storage. Transfusion 60, 1212-1226. doi: 10.1111/trf.15810

Brand, M. D. (2016). Mitochondrial generation of superoxide and hydrogen peroxide as the source of mitochondrial redox signalling. Free Radic. Biol. Med. 100, 14-31. doi: 10.1016/j.freeradbiomed.2016.04.001

da Silva, L. A., Tromm, C. B., Bom, K. F., Mariano, I., Pozzi, B., da Rosa, G. L., et al. (2014). Effects of taurine supplementation following eccentric exercise in young adults. Appl. Physiol. Nutr. Metab. 39, 101-104. doi: 10.1139/apnm-2012-0229

Dawson, R. Jr., Biasetti, M., Messina, S., and Dominy, J. (2000). The cytoprotective role of taurine in exercise-induced muscle injury. Amino Acids 22, 309-324. doi: $10.1007 /$ s007260200017

Dawson, R. Jr., Biasetti, M., Messina, S., and Dominy, J. (2002). The cytoprotective role of taurine in exercise-induced muscle injury. Amino Acids 22, 309-324.

De Carvalho, F. G., Galan, B. S. M., Santos, P. C., Pritchett, K., Pfrimer, K., Ferriolli, E., et al. (2017). Taurine: a potential ergogenic aid for preventing muscle damage and protein catabolism and decreasing oxidative stress produced by endurance exercise. Front. Physiol. 8:710. doi: 10.3389/fphys.2017.00710

De Luca, A., Pierno, S., and Camerino, D. C. (2015). Taurine: the appeal of a safe amino acid for skeletal muscle disorders. J. Transl. Med. 13:243. doi: 10.1186/ s12967-015-0610-1

De Luca, A., Pierno, S., Liantonio, A., Cetrone, M., Camerino, C., Fraysse, B., et al. (2003). Enhanced dystrophic progression in $\mathrm{mdx}$ mice by exercise and beneficial effects of taurine and insulin like growth factor-1. J. Pharmacol. Exp. Ther. 304, 453-463. doi: 10.1124/jpet.102.041343

El Idrissi, A. (2008). Taurine increases mitochondrial buffering of calcium: role in neuroprotection. Amino Acids 34, 321-328. doi: 10.1007/s00726-006-0396-9

Faggiano, A., Melis, D., Alfieri, R., De Martino, M., Filippella, M., Milone, F., et al. (2005). Sulfur amino acids in Cushing's disease: insight in homocysteine and taurine levels in patients with active and cured disease. J. Clin. Endocrinol. Metab. 90, 6616-6622. doi: 10.1210/jc.2005-0656

Flann, K. L., LaStayo, P. C., McClain, D. A., Hazel, M., and Lindstedt, S. L. (2011). Muscle damage and muscle remodeling: no pain, no gain? J. Exp. Biol. 214, 674-679. doi: 10.1242/jeb.050112 with taurine supplementation must be established in both human and animal models.

\section{AUTHOR CONTRIBUTIONS}

AT, RP, JB, BI, and YG conceived the idea and wrote the manuscript. All the authors contributed to the final version of the manuscript.

\section{ACKNOWLEDGMENTS}

The authors would like to acknowledge Mr. Kishore Kumar who helped in editing the manuscript.

Frontera, W. R., and Ochala, J. (2015). Skeletal muscle: a brief review of structure and function. Calcif. Tissue Int. 96, 183-195. doi: 10.1007/s00223-014-9915-y

Galan, B. S., Carvalho, F. G., Santos, P. C., Gobbi, R. B., Kalva-Filho, C. A., Papoti, M., et al. (2018). Effects of taurine on markers of muscle damage, inflammatory response and physical performance in triathletes. J. Sports. Med. Phys. Fitness 58, 1318-1324. doi: 10.23736/S0022-4707.17.07497-7

Galloway, S. D., Talanian, J. L., Shoveller, A. K., Heigenhauser, G. J., and Spriet, L. L. (2008). Seven days of oral taurine supplementation does not increase muscle taurine content or alter substrate metabolism during prolonged exercise in humans. J. Appl. Physiol. 105, 643-651. doi: 10.1152/japplphysiol.90525.2008

Goodman, C. A., Horvath, D., Stathis, C., Mori, T., Croft, K., Murphy, R. M., et al. (2009). Taurine supplementation increases skeletal muscle force production and protects muscle function during and after high-frequency in vitro stimulation. J. Appl. Physiol. 107, 144-154. doi: 10.1152/japplphysiol.00040.2009

Hansen, S. H., Andersen, M. L., Cornett, C., Gradinaru, R., and Grunnet, N. (2010). A role for taurine in mitochondrial function. J. Biomed. Sci. 17:S23. doi: 10.1186/1423-0127-17-S1-S23

Harty, P. S., Cottet, M. L., Malloy, J. K., and Kerksick, C. M. (2019). Nutritional and supplementation strategies to prevent and attenuate exercise-induced muscle damage: a brief review. Sports Med. Open 5:1. doi: 10.1186/s40798-018-0176-6

Hood, D. A., Memme, J. M., Oliveira, A. N., and Triolo, M. (2019). Maintenance of skeletal muscle mitochondria in health, exercise, and aging. Annu. Rev. Physiol. 81, 19-41. doi: 10.1146/annurev-physiol-020518-114310

Horvath, D. M., Murphy, R. M., Mollica, J. P., Hayes, A., and Goodman, C. A. (2016). The effect of taurine and beta-alanine supplementation on taurine transporter protein and fatigue resistance in skeletal muscle from mdx mice. Amino Acids 48, 2635-2645. doi: 10.1007/s00726-016-2292-2

Husain, N., and Mahmood, R. (2020). Taurine attenuates Cr(VI)-induced cellular and DNA damage: an in vitro study using human erythrocytes and lymphocytes. Amino Acids 52, 35-53. doi: 10.1007/s00726-019-02807-1

Huxtable, R. J. (1992). Physiological actions of taurine. Physiol. Rev. 72, 101-163. doi: 10.1152/physrev.1992.72.1.101

Huxtable, R. J. (2000). Expanding the circle 1975-1999: sulfur biochemistry and insights on the biological functions of taurine. Adv. Exp. Med. Biol. 483, 1-25.

Ince, S., Arslan-Acaroz, D., Demirel, H. H., Varol, N., Ozyurek, H. A., Zemheri, F., et al. (2017). Taurine alleviates malathion induced lipid peroxidation, oxidative stress, and proinflammatory cytokine gene expressions in rats. Biomed. Pharmacother. 96, 263-268. doi: 10.1016/j.biopha.2017.09.141

Ito, T., Oishi, S., Takai, M., Kimura, Y., Uozumi, Y., Fujio, Y., et al. (2010). Cardiac and skeletal muscle abnormality in taurine transporter-knockout mice. J. Biomed. Sci. 17:S20. doi: 10.1186/1423-0127-17-S1-S20

Ito, T., Yoshikawa, N., Schaffer, S. W., and Azuma, J. (2014). Tissue taurine depletion alters metabolic response to exercise and reduces running capacity in mice. J. Amino Acids 2014:964680. doi: 10.1155/2014/964680

Kawamura, T., and Muraoka, I. (2018). Exercise-induced oxidative stress and the effects of antioxidant intake from a physiological viewpoint. Antioxidants 7:119. doi: 10.3390/antiox7090119

Kim, C., and Cha, Y. N. (2009). "Production of reactive oxygen and nitrogen species in phagocytes is regulated by taurine chloramine," in Taurine 7. Advances in 
Experimental Medicine and Biology, eds J. Azuma, S. W. Schaffer, and T. Ito (New York, NY: Springer).

Kim, C., Jang, J. S., Cho, M. R., Agarawal, S. R., and Cha, Y. N. (2010). Taurine chloramine induces heme oxygenase- 1 expression via $\mathrm{Nrf} 2$ activation in murine macrophages. Int. Immunopharmacol. 10, 440-446. doi: 10.1016/j.intimp.2009. 12.018

Kolossov, V. L., Beaudoin, J. N., and PrabhuPonnuraj, N. (2015). Thiol-based antioxidants elicit mitochondrial oxidation via respiratory complex III. Am. J. Physiol. Cell Physiol. 309, C81-C91. doi: 10.1152/ajpcell.00006.2015

Lambert, I. H., Kristensen, D. M., Holm, J. B., and Mortensen, O. H. (2015). Physiological role of taurine-from organism to organelle. Acta Physiol. 213, 191-212. doi: 10.1111/apha.12365

Li, S., Wei, B. K., Wang, J., Dong, G., and Wang, X. (2019). Taurine supplementation ameliorates arsenic-induced hepatotoxicity and oxidative stress in mouse. Adv. Exp. Med. Biol. 1155, 463-470. doi: 10.1007/978-981-138023-5_43

Lin, S., Hirai, S., Yamaguchi, Y., Goto, T., Takahashi, N., Tani, F., et al. (2013). Taurine improves obesity-induced inflammatory responses and modulates the unbalanced phenotype of adipose tissue macrophages. Mol. Nutr. Food. Res. 57, 2155-2165. doi: 10.1002/mnfr.201300150

Maleki, V., Mahdavi, R., Hajizadeh-Sharafabad, F., and Alizadeh, M. (2020). The effects of taurine supplementation on oxidative stress indices and inflammation biomarkers in patients with type 2 diabetes: a randomized, double-blind, placebo-controlled trial. Diabetol. Metab. Syndr. 12:9. doi: 10.1186/s13098-0200518-7

Matsuzaki, Y., Miyazaki, T., Miyakawa, S., Bouscarel, B., Ikegami, T., and Tanaka, N. (2002). Decreased taurine concentration in skeletal muscles after exercise for various durations. Med. Sci. Sports Exerc. 34, 793-797. doi: 10.1097/00005768200205000-00011

McLeay, Y., Stannard, S., and Barnes, M. (2017). The effect of taurine on the recovery from eccentric exercise-induced muscle damage in males. Antioxidants 6:79. doi: 10.3390/antiox6040079

Miyazaki, T., Honda, A., Ikegami, T., and Matsuzaki, Y. (2013). The role of taurine on skeletal muscle cell differentiation. Adv. Exp. Med. Biol. 776, 321-328. doi: 10.1007/978-1-4614-6093-6029

Molnár, A., JónásnéSztruhár, I., Csontos, ÁA., Ferencz, C., Várbíró, S., and Székács, B. (2016). Special nutrition intervention is required for muscle protective efficacy of physical exercise in elderly people at highest risk of sarcopenia. Physiol. Int. 103, 368-376. doi: 10.1556/2060.103.2016.3.12

Mozaffari, M. S., Tan, B. H., Lucia, M. A., and Schaffer, S. W. (1986). Effect of druginduced taurine depletion on cardiac contractility and metabolism. Biochem. Pharmacol. 35, 985-989. doi: 10.1016/0006-2952(86)90087-0

Nam, S. Y., Kim, H. M., and Jeong, H. J. (2017). The potential protective role of taurine against experimental allergic inflammation. Life Sci. 184, 18-24. doi: 10.1016/j.lfs.2017.07.007

Neubauer, O., Reichhold, S., Nersesyan, A., König, D., and Wagner, K. H. (2008). Exercise-induced DNA damage: is there a relationship with inflammatory responses? Exerc. Immunol. Rev. 14, 51-72.

Niu, X., Zheng, S., Liu, H., and Li, S. (2018). Protective effects of taurine against inflammation, apoptosis, and oxidative stress in brain injury. Mol. Med. Rep. 18, 4516-4522. doi: 10.3892/mmr.2018.9465

Pastukh, V., Ricci, C., Solodushko, V., Mozaffari, M., and Schaffer, S. W. (2005). Contribution of the PI 3-kinase/Akt survival pathway toward osmotic preconditioning. Mol. Cell. Biochem. 269, 59-67. doi: 10.1007/s11010-0052536-z

Qin, F., Siwik, D. A., Lancel, S., Zhang, J., Kuster, G. M., Luptak, I., et al. (2013). Hydrogen peroxide-mediated SERCA cysteine 674 oxidation contributes to impaired cardiac myocyte relaxation in senescent mouse heart. J. Am. Heart Assoc. 2:e000184. doi: 10.1161/JAHA.113.000184

Ra, S. G., Choi, Y., Akazawa, N., Ohmori, H., and Maeda, S. (2016). Taurine supplementation attenuates delayed increase in exercise-induced arterial stiffness. Appl. Physiol. Nutr. Metab. 41, 618-623. doi: 10.1139/apnm-20150560

Radák, Z., Taylor, A. W., Ohno, H., and Goto, S. (2001). Adaptation to exerciseinduced oxidative stress: from muscle to brain. Exerc. Immunol. Rev. 7, 90-107.

Rahman, M. M., Park, H. M., Kim, S. J., Go, H. K., Kim, G. B., Hong, C. U., et al. (2011). Taurine prevents hypertension and increases exercise capacity in rats with fructose-induced hypertension. Am. J. Hypertens. 24, 574-581. doi: 10.1038/ajh.2011.4

Rivas, D. A., and Fielding, R. A. (2012). Skeletal Muscle. In Encyclopedia of Human Nutrition. Cambridge, MA: Academic Press.

Sajid, I., Ahmad, S., Emad, S., Batool, Z., Khaliq, S., Anis, L., et al. (2017). Enhanced physical endurance and improved memory performance following taurine administration in rats. Pak. J. Pharm. Sci. 30, 1957-1963.

Schaffer, S. W., Jong, C. J., Ramila, K. C., and Azuma, J. (2010). Physiological roles of taurine in heart and muscle. J. Bio.med. Sci. 17:S2. doi: 10.1186/1423-012717-S1-S2

Schaffer, S. W., Shimada, K., Jong, C. J., Ito, T., Azuma, J., and Takahashi, K. (2014). Effect of taurine and potential interactions with caffeine on cardiovascular function. Amino Acids 46, 1147-1157. doi: 10.1007/s00726-014-1708-0

Schaffer, S. W., Solodushko, V., and Kakhniashvili, D. (2002). Beneficial effect of taurine depletion on osmotic sodium and calcium loading during chemical hypoxia. Am. J. Physiol. Cell Physiol. 282, C1113-C1120. doi: 10.1152/ajpcell. 00485.2001

Scheele, C., Nielsen, S., and Pedersen, B. K. (2009). ROS and myokines promote muscle adaptation to exercise. Trends Endocrinol. Metab. 20, 95-99. doi: 10. 1016/j.tem.2008.12.002

Seidel, U., Huebbe, P., and Rimbach, G. (2019). Taurine: a regulator of cellular redox homeostasis and skeletal muscle function. Mol. Nutr. Food Res. 63:e1800569. doi: 10.1002/mnfr.201800569

Silva, L. A., Silveira, P. C., Ronsani, M. M., Souza, P., Scheffer, D., Vieira, L., et al. (2011). Taurine supplementation decreases oxidative stress in skeletal muscle after eccentric exercise. Cell. Biochem. Funct. 29, 43-49. doi: 10.1002/cbf.1716

Silveira, P. C., da Silva, L. A., Pinho, C. A., De Souza, P. S., Ronsani, M. M., Scheffer, D. L., et al. (2013). Effects of low-level laser therapy (GaAs) in an animal model of muscular damage induced by trauma. Lasers Med. Sci. 28, 431-436. doi: 10.1007/s10103-012-1075-6

Soares, J. P., Silva, A. M., Oliveira, M. M., Peixoto, F., Gaivão, I., and Mota, M. P. (2015). Effects of combined physical exercise training on DNA damage and repair capacity: role of oxidative stress changes. Age 37:9799. doi: 10.1007/ s11357-015-9799-4

Sugiura, H., Okita, S., Kato, T., Naka, T., Kawanishi, S., Ohnishi, S., et al. (2013). Protection by taurine against INOS-dependent DNA damage in heavily exercised skeletal muscle by inhibition of the NF-кBsignaling pathway. Adv. Exp. Med. Biol. 775, 237-246. doi: 10.1007/978-1-4614-6130-2_20

Suhr, F., Gehlert, S., Grau, M., and Bloch, W. (2013). Skeletal muscle function during exercise-fine-tuning of diverse subsystems by nitric oxide. Int. J. Mol. Sci. 14, 7109-7139. doi: 10.3390/ijms14047109

Sun Jang, J., Piao, S., Cha, Y. N., and Kim, C. (2009). Taurine chloramine activates Nrf2, increases HO-1 expression and protects cells from death caused by hydrogen peroxide. J. Clin. Biochem. Nutr. 45, 37-43. doi: 10.3164/jcbn. 08-262

Terrill, J. R., Pinniger, G. J., Graves, J. A., Grounds, M. D., and Arthur, P. G. (2016). Increasing taurine intake and taurine synthesis improves skeletal muscle function in the mdx mouse model for Duchenne muscular dystrophy. J. Physiol. 594, 3095-3110. doi: 10.1113/JP271418

Thirupathi, A., Freitas, S., Sorato, H. R., Pedroso, G. S., Effting, P. S., Damiani, A. P., et al. (2018). Modulatory effects of taurine on metabolic and oxidative stress parameters in a mice model of muscle overuse. Nutrition 54, 158-164. doi: 10.1016/j.nut.2018.03.058

Thirupathi, A., and Pinho, R. A. (2018). Effects of reactive oxygen species and interplay of antioxidants during physical exercise in skeletal muscles. J. Physiol. Biochem. 74, 359-367. doi: 10.1007/s13105-018-0633-1

Thirupathi, A., Pinho, R. A., and Chang, Y. Z. (2020). Physical exercise: an inducer of positive oxidative stress in skeletal muscle aging. Life Sci. 252:117630. doi: 10.1016/j.lfs.2020.117630

Uozumi, Y., Ito, T., Hoshino, Y., Mohri, T., Maeda, M., Takahashi, K., et al. (2006). Myogenic differentiation induces taurine transporter in association with taurine-mediated cytoprotection in skeletal muscles. Biochem. J. 394, 699-706. doi: 10.1042/BJ20051303

Vaanholt, L. M., Speakman, J. R., Garland, T. Jr., Lobley, G. E., and Visser, G. H. (2008). Protein synthesis and antioxidant capacity in aging mice: effects of long-term voluntary exercise. Physiol. Biochem. Zool. 81, 148-157. doi: 10.1086/ 525289 
Waldron, M., Patterson, S. D., and Jeffries, O. (2019). Oral taurine improves critical power and severe-intensity exercise tolerance. Amino Acids 51, 1433-1441. doi: 10.1007/s00726-019-02775-6

Ward, R., Bridge, C. A., McNaughton, L. R., and Sparks, S. A. (2016). The effect of acute taurine ingestion on 4-km time trial performance in trained cyclists. Amino Acids 48, 2581-2587. doi: 10.1007/s00726-016-2282-4

Ward, R. J., Francaux, M., Cuisinier, C., Sturbois, X., and De Witte, P. (1999). Changes in plasma taurine levels after different endurance events. Amino Acids 16, 71-77. doi: 10.1007/BF01318886

Warskulat, U., Flogel, U., Jacoby, C., Hartwig, H. G., Thewissen, M., Merx, M. W., et al. (2004). Taurine transporter knockout depletes muscle taurine levels and results in severe skeletal muscle impairment but leaves cardiac function uncompromised. FASEB J. 18, 577-579. doi: 10.1096/fj.030496fje

Waters, D. L., Baumgartner, R. N., Garry, P. J., and Vellas, B. (2010). Advantages of dietary, exercise-related, and therapeutic interventions to prevent and treat sarcopenia in adult patients: an update. Clin. Interv. Aging 5, 259-270. doi: $10.2147 /$ cia.s6920
Yatabe, Y., Miyakawa, S., Miyazaki, T., Matsuzaki, Y., and Ochiai, N. (2003). Effects of taurine administration in rat skeletal muscles on exercise. J. Orthop. Sci. 8, 415-419. doi: 10.1007/s10776-002-0636-1

Zhang, M., Izumi, I., Kagamimori, S., Sokejima, S., Yamagami, T., Liu, Z., et al. (2004). Role of taurine supplementation to prevent exercise-induced oxidative stress in healthy young men. Amino Acids 26, 203-207. doi: 10.1007/s00726003-0002-3

Conflict of Interest: The authors declare that the research was conducted in the absence of any commercial or financial relationships that could be construed as a potential conflict of interest.

Copyright (C) 2020 Thirupathi, Pinho, Baker, István and Gu. This is an open-access article distributed under the terms of the Creative Commons Attribution License (CC BY). The use, distribution or reproduction in other forums is permitted, provided the original author(s) and the copyright owner(s) are credited and that the original publication in this journal is cited, in accordance with accepted academic practice. No use, distribution or reproduction is permitted which does not comply with these terms. 